\title{
XXVI. Some lecture experiments illustrating syntony
}

\section{Philip E. Shaw B.A. B.Sc.}

To cite this article: Philip E. Shaw B.A. B.Sc. (1900) XXVI. Some lecture experiments illustrating syntony, Philosophical Magazine Series 5, 50:304, 283-290, DOI: $\underline{10.1080 / 14786440009463915}$

To link to this article: http://dx.doi.org/10.1080/14786440009463915

曲 Published online: 21 Apr 2009.

Submit your article to this journal $\pi$

Џll Article views: 3

Q View related articles $\sqsubset$ 
of the tube. The electric force in the striated part of the discharge is much less than that close to the cathode; and it might be urged that if such an intense field were required to ionize the gas close to the cathode, the smaller field in the striated discharge would not produce ionization. It must, however, be remembered that the ionization at the cathode has to be produced by positive ions, while that in the rest of the field may be due to negative corpuscles ; and it may very well be the case that the large positive ions are less efficient as ionizers than the negative corpuscles.

XXVI. Some Lecture Experiments illustrating Syntony. By Philit E. Shaw, B.A., B.Sc.*

T $N$ the Journal of the Institution of Electrical Engineers (Feb. 1899; will be found an account by Prof. Oliver Jodge of his researches in Magnetic Space Telegraphy. The present writer then set up some apparatus to show these methods in a popular lecture. Considerable difficulties soon presented themselves, but success was ultimately attained. Those who are conversant with the above paper will adnit that the theory involved in it is of importance, and that any new demonstration of it would be valuable.

Besides the interest attaching to the theory of syntony, this method of magnetic space-telegraphy has promise of practical and commercial value, and has been shown already by Prof. Lodge to work well over considerable distances.

The following brief account contains a notice of the general method, of special details introduced by the author, and of precautions to be adopted.

\section{Theory.}

A summary will be given of the more essential points.

I. The frequency $(N)$ of a circuit of capacity $S$ and selfinduction $\mathrm{L}$ is

$$
\mathrm{N}=\frac{160}{\sqrt{\mathrm{L} \cdot \mathrm{s}}}
$$

where $\mathrm{L}$ is reckoned in henries and $\mathrm{S}$ in microfarads.

II. The mutual induction of two parallel coaxial coils of turns $n$ and $m$, radii $a$ and $b$, at distance $r$ apart is, assuming $r$ large,

$$
\mathrm{M}=\frac{m \pi a^{2} \cdot n \pi b^{2}}{r^{3}}
$$

* Cormunicated by the Physical Society : read March 23, 1900. 
III. The self-induction of a short flat coil, as used for sending or receiving, is

$$
\mathrm{L}=4 \pi \mu n^{2} a\left(\log _{e} \frac{8 a}{c}-2\right),
$$

and for a long solenoid (neglecting a small correction for the ends)

$$
\begin{gathered}
\mathrm{L}=\frac{4 \pi \mu n^{2} \mathrm{~A}}{\ell} . \\
\left\{\begin{array}{l}
n=\text { number of turns; } \\
a=\text { radius of coil } ; \\
c=\text { radius of wire; } \\
\mathrm{L}=\text { length of solenoid; } \\
\mathrm{A}=\text { mean sectional area. }
\end{array}\right. \\
\text { IV. Current Amplitude }=\frac{\mathrm{\textrm {E } _ { 0 } / \mathrm { R }}}{\sqrt{1+\frac{\mathrm{L}}{\mathrm{SR}^{2}}\left(\frac{p}{p_{0}}-\frac{p_{0}}{p}\right)^{2}}}, \\
\text { where } \frac{p_{0}}{2 \pi}=\text { natural frequency of circuit, } \\
\frac{p}{2 \pi}=\text { frequency of alternating-current in circnit; }
\end{gathered}
$$

but for exact resonance, the current-amplitude $=E_{0} / R$; hence for sharp resonance (since then $p=p_{0}$ ), $\frac{\mathrm{L}}{\mathrm{SH}^{2}}$ must be large.

V. Magnification Ratio, due to resonance, is given by the expression

$$
\frac{\mathrm{V}}{\mathrm{E}_{0}}=\frac{p \mathrm{I}_{4}}{\mathrm{R}},
$$

supposing the damping is small ;

where $\mathrm{E}_{0}=$ impressed E.M.F. from current source,

$\mathrm{V}=$ effective potential-difference at the condenserterminals.

Now we have as far as possible to arrange the circuits in accordance with the above principles, and where these seem mutually in opposition to make a judicious compromise.

The desiderata are:-

(1) $\mathbb{R}$ small, to obtain large current-amplitude, small damping, sharp resonance, and large magnificationratio. Notice that $R$ includes hysteresis if an iron core is used, and eddy-currents in neighbouring circuits. 
(2) L large, to obtain sharp resonance and large magnifcation-ratio.

(3) S small, to obtain sharp resonance and to avoid large sparks at ' make' and 'break.'

(4) Radius of sending and receiving coils large, to obtain large $\mathrm{N}$.

(5) The auxiliary coils must be suited to conditions (1) and (2), othorwise they may have any size or shape. For the comparatively small frequency used, the selfinduction part of the impedance need not be considered.

\section{The Circuits.}

The sending-circuit is shown in the sketch (fig. 1 ), which is in elevation. The current leaving the cells at the top end

Fig. 1.

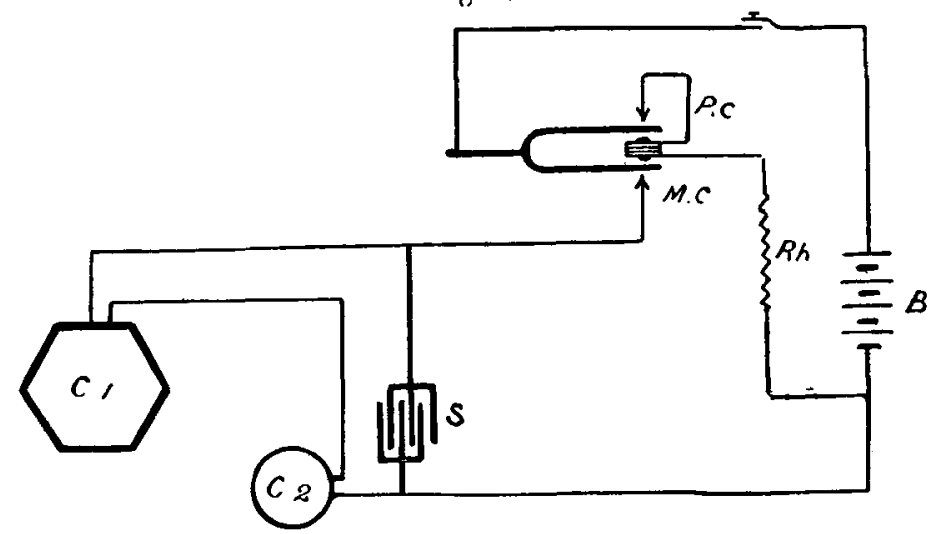

passes through the key, fork, platinum-contact (P.C.), rheostat, and to the lower end of the cells; the fork is worked by the passage of this clirrent through the electromagnet between the prongs of the fork.

But there is another branch to the circuit, beginning at the mercury-contact (M.C.), then through the coils $\mathrm{C}_{1}$ and $\mathrm{C}_{2}$, the adjustable condenser $\mathrm{S}$ being put as a shunt to the two coils, then to the lower end of the cells.

Thus if $\mathrm{S}$ were removed we should obtain current-alternations in the coils of the frequency (100) of the fork; but with $S$ in place, we have a frequency depending on the values of $L$ and $S$ in the coils. In setting up the system we select suitable coils and condenser, calculating the values of $\mathrm{L}$ from the dimensions of the coils; then put in such a value of

Phil. Mag. S. 5. Vol. 50. No. 304. Sept. $1900 . \quad$ X 
$\mathrm{S}$ as will give the required frequency, and finally adjust to a maximum by the iron core in $\mathrm{C}_{2}$ (see expression I.).

This frequency ( 400 was used) must be an exact multiple of that of the fork (100), then between each charge received by $\mathrm{S}$ from the fork, there would be four complete surgings between $\mathrm{S}, \mathrm{C}_{1}, \mathrm{C}_{2}$; the potential changes would be considerable, and violent shocks might be obtained.

L may be varied by the introduction of any number of coils at $\mathrm{C}_{2}$, or by moving a core of iron wires in $\mathrm{C}_{2}$. This latter is a convenient adjustment, but wasteful on account of bysteresis. The adjustments are (1) the mercury-contact (M.C.), (2) the rheostat, (3) adjustable $\mathrm{L}$ in $\mathrm{C}_{2}$. Place one ear on the flat condenser (taking care to avoid getting a shock from its terminals), listen to the note heard in it, and change (1), (2), and (3) up to maximum loudness. The adjustment (1) must on no account be neglected ; the method adopted was to have the fork rigidly held, but capable of being rotated by any small amount in a vertical plane.

Thus, as above described, we have a circuit with a given frequency, whose note can be raised to a maximum intensity. Now turn to the secondary (or receiving) circuit (fig. 2).

Fig. 2.

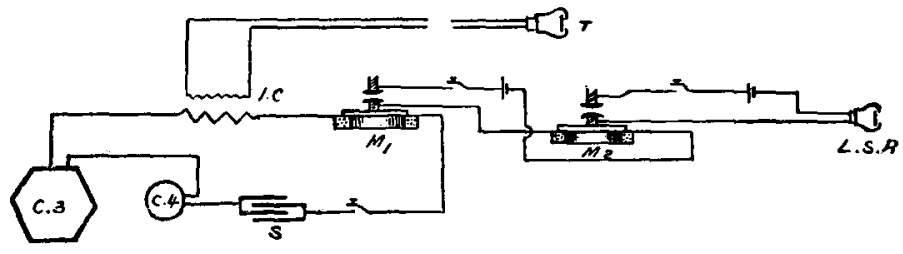

The coil $\mathrm{C}_{3}$ is placed parallel to $\mathrm{C}_{1}$, and catches the waves from it; $\mathrm{C}_{4}$ is an auxiliary coil. The condenser $\mathrm{S}$ is in series in the circuit, and not, as in the primary, shunted across the ends of the coils. There are two other coils in the same circuit:-(1) $M_{1}$ is the coil of the relay, with its microphonecarbons shown above it ; (2) the small resistance-winding of a telephone induction-coil, the large resistance-winding being in series with the telephone $\mathrm{T}$.

If now the coil $\mathrm{C}_{3}$ is taking up the waves from the sendingcircuit, and the variables $\mathrm{C}_{4}$ and $\mathrm{S}$ are set for exact tuning (to frequency 400) a note of maximum loudness will be heard in the telephone T. In the primary it is convenient, though wasteful, to have an iron core in a coil for the small adjustment of $L$, but in the secondary it would be useless, since $\mu$ is small for the very weak currents existing. 
Now put in the first relay by adjusting the carbons at $M_{1}$. The action occurring at $\mathbf{M}_{1}$ and $\mathbf{M}_{2}$ must be explained in detail. The coil $\mathrm{M}_{1}$ lies in a horizontal annular space in which the lines of force run radially, and normal to the wires in the coil ; then if current-alternations occur in the coil, it is impelled up and down with the frequency of the alternations; the field meanwhile is maintained constant by a separate strong current (say 10 amps.). The movements of the coil will, by the action of the microphone, produce alternating currents of a higher order of intensity in the relay-coil $M_{2}$; and this, in turn, will in like manner give strong currents in the last eircuit, producing a loud note in the loud-speaking receiver (L.S.R.). Although, to avoid confusion in the diagram, no such connexions are shown, it is easy to put the induction-coil and telephone (I.C. and T.) into the second circuit, and by them adjust the working of $M_{1}$ to a maximum intensity.

The relays should be guarded from all vibrations by being put on blocks resting on soft rubber balls ; also they should be on separate blocks, otherwise the action of one will start the other.

Description of the Relay.

The principle involved is due to Prof. Lodge, and is found in his magnifying-telephone; but the attachments of the coil and the system suspending it are due to the author, and will be shortly described.

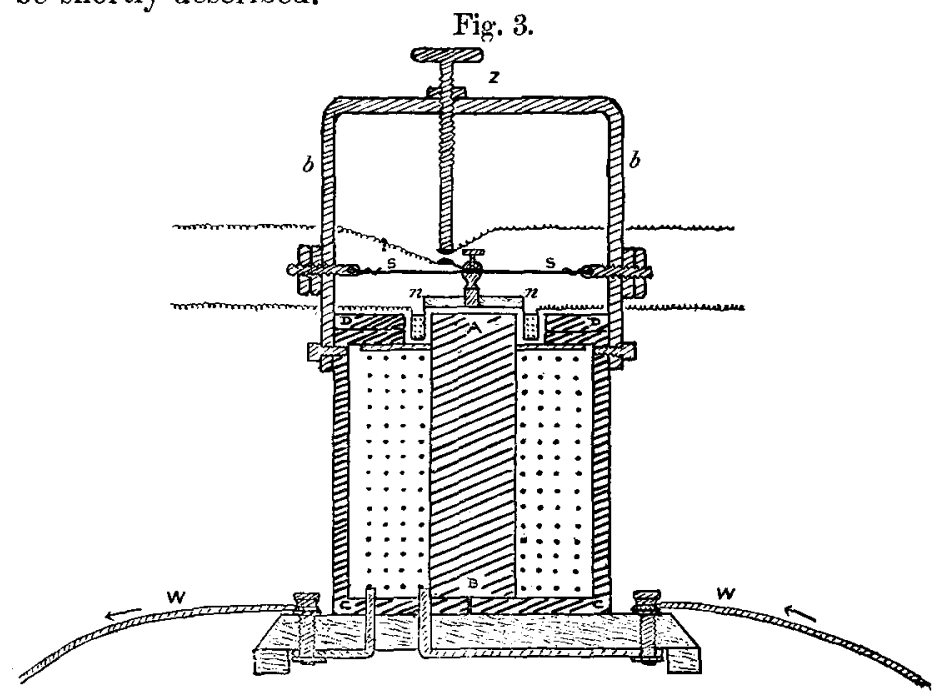

The wires WW (fig. 3) carry the strong current (say 10 amps.) used to produce a field ; the magnetic circuit of $\mathrm{X} 2$ 
soft iron is seen to be ABCDA; it has the air-gap between $\mathrm{D}$ and $\mathrm{A}$ in which the coil $n n$ lies. This is the coil $\mathrm{M}_{1}$ or $\mathrm{M}_{2}$ in fig. 2.

The coil $n n$ is very light, 60 turns of covered copper wire ( 36 s.w.G.), resistance about 4 ohms, wound on a paper vill-box, which is light and rigid and perfectly circular, and is hence a very convenient former for the coil. The steel wire $s s$ has a light brass fitting screwed tightly to its centre; below this is a light bow of cedar lashed and cemented to the brass ahove and lashed and cemented to the coil below.

As seen in the sketch, the wire is fixed (bent on itself and brazed) to a brass screw at each end, each provided with two nuts, on the outside of the stout brass frame $b b$.

By tightening the wire we can produce in it the required frequency, 400 (this can be observed by gently tapping the wire and listening for its natural frequency); then alternations in the current of $n n$ must produce vibration in the wire $s s$, and when the carbons are set by the screw $z$ we have the microphono acting.

Two graphite buitons, or one graphite and one hard carbon button, were found to act very well.

Another form of relay was used. Take a Blake transmitter, change the stiff' iron plate for a more yielding one, and attach the coil $n n$ to it. This acts fairly, but is obviously not syntonic with the whole system as in the other case.

\section{Manipulation.}

The chief special difficulties may be mentioned categorically.

I. It must be borne in mind that in such an apparatus as that used, where the ratio $\frac{R}{\mathrm{~L}}$ is comparatively large, damping occurs rapidly, and consequently the current-amplitude dies out rapidly between each fresh charge received from the fork.

Fig. 4.

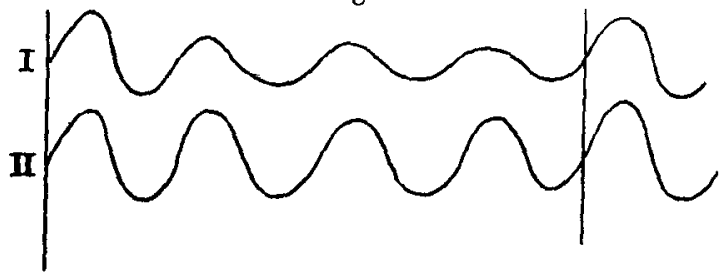

We supposed the fork to have frequency 100 , and the circuit to hare frequency 400 . Then the curve for current would be of the nature shown in I.; whereas the ideal curve, where $\frac{R}{L}$ is negligible, would be as in II., where the amplitude is 
seen to remain constant. Consequently in our system we should have a pronounced fundamental 100 as well as an harmonic 400 , and we have to learn to pick out the latter and, in adjusting the circuit, to work it up to a maximum. This applies to both primary and secondary circuits.

II. Care must be taken to properly adjust the platinum and mercury contacts, not only to produce the maximum true sound in the circuit, but also to avoid any spurting at the contact; this would have its counterpart in violent spurts in the sounds in the coils, which mask the true effect.

III. The greatest difficulty experienced, however, was in the microphones, both (1) in obtaining them truly syntonic with the circuit and with one another, and (2) in the minute adjustment of the earbons for very weak currents. The special relay described above worked well, but requires delicate handling in respect of both (1) and (2). Anyone who has worked a granular transmitter, or indeed any microphone with a view to obtaining maximum effects for very small movements, will admit that an exceedingly fine arjustment is required. This might seem to be self-evident, but the author has made independent research on this point, his results being that a movement of one carbon of the order $10^{-5} \mathrm{~cm}$. would carry the microphone from hard contact (no microphone action) to complete freedom. Hence the difficulty above stated is quite intelligible.

In working the relay described previously, the final adjustment was made by gently pressing with the finger on the stout brass bar 67 (the section of the bar is $\frac{1}{2}$ in. $\times \frac{1}{4}$ in.). This of itself indicates the delicacy of the microphone.

IV. The design of the sending and receiving coils. The author had in each of them 100 turns of insulated copper wire (16 s.w.G.), radius about half a metre, resistance about 3 ohms. If the same wire had been opened out to 50 turns of radius one metre in both sender and receiver, the mutual induction would be four times as great ; the self-induction of each would be slightly reduced (roughly to about two-thirds of its former value), but this could be made up in the anxiliary coils. Thus it is advantageous to have the radius of the coils large. In making the auxiliary coils we require to have large $L$ with small $R$. If we have a given amount of wire at our disposal, it is better to have small radius and many turns, and have a core of iron wires for final adjustment. One advantage of keeping $R$ moderately large in the circuit is that we run no risk of breaking down the condenser in the primary.

V. In Prof. Lodge's research the sound produced at the 
receiving-telephone was very small, just sufficient for audibility being all that is required by a single observer ; but in the case here discussed, we have to produce sounds to be given out to a large audience by a loud-speaking telephone ; these would have to be, and were, thousands of times as great as required in the former case. The process of amplifying the small currents until they become large and produce really loud signals is a difficult one in itself; but an even greater one arises from the passage of large currents through the last microphone; the carbon-contacts are rapidly burnt away, and as a microphone contact is a very delicate one, continual adjustment is required.

To meet this special difficulty Prof. Lodge has employed an ingenious device which should be mentioned. The upper carbon is attached to a large mass which turns freely on a horizontal axis, which is so counterpoised that the two carbons are gently but steadily pressed together. If the carbons burn away at the contact, the piroted system moves on its axis and maintains the same microphone-action as before. Thus the contact is kept automatically in working order.

It should be pointed out that though the sounds produced in the receiver were much louder than in the case of Prof. Lodge's system, yet the distance between sender and receiver was in his case very much larger; so that each arrangement has its own difficulties.

\section{Another Experiment.}

By a small modification in the primary circuit, tuning can be demonstrated in a very striking way. In fig. 1 put a loud-speaking receiver in series with the coils $\mathrm{C}_{1}$ and $\mathrm{C}_{2}$, and shunt it by a resistance-box. On working the circuit this receiver gives out a loud sound of the circuit-frequency; by changes in the shunt we can affect its intensity, while by changes in $\mathrm{L}$ or $\mathrm{C}$ we alter its frequency. It may be mentioned in passing that this is a convenient means of producing a sound of constant intensity and is easily adjustable (though not necessarily pure), and as such it has been used by the author.

As a simple example, if we change the capacity from $0.9 \mathrm{mfd}$. to $0.4 \mathrm{mfd}$., then the frequency should change in the ratio $\sqrt{\overline{9}}=\frac{2}{3}$; the notes will be found to have about a musical interval of one-fifth.

The author's best thanks are due to Prof. Lodge for advice during the progress of the work. 